\title{
Effectiveness and safety of sulodexide in the treatment of venous diseases
}

\author{
Witold Tomkowski, Małgorzata Dybowska \\ National Institute of Tuberculosis and Respiratory Disease, Warsaw, Poland
}

\begin{abstract}
This article presents a review of the literature assessing the effectiveness and safety of sulodexide in the prophylaxis of deep vein thrombosis and treatment of chronic venous disease. It was demonstrated that sulodexide is effective and safe in the prophylaxis of deep vein thrombosis and treatment of chronic venous insufficiency with ulcers. Sulodexide is characterized by low frequency of bleeding complications.
\end{abstract}

Key words: sulodexide, venous thromboembolic disease, chronic venous disease

Acta Angiol 2019; 25, 3: 157-161

Sulodexide is a naturally occurring glycosaminoglycan obtained from pig intestine. Clinical uses of sulodexide include prevention of venous thromboembolic disease (VTD) recurrence, treatment of chronic venous disease (CVD) and moderate chronic arterial occlusive disease of the lower limbs. In this publication, we analyze the scientific evidence regarding effectiveness and safety of sulodexide in the treatment of VTD and CVD.

In order to understand the pharmacological functions of sulodexide, it is necessary to know the structure of vascular endothelium. The surface of tunica intima of those vessels is lined by glycocalyx, which is composed of proteoglycans, hyaluronic acid, glycoproteins, and white cells absorbed on its surface. Glycocalyx plays a number of important roles: regulates vascular permeability, controls their tone, determines interaction of vascular wall with leukocytes and thrombocytes, and regulates response to vascular stress. The above-mentioned mechanisms are decisive for proper blood flow, which is directly related to anticoagulant and pro-fibrinolytic action of glycocalyx [I, 2]. Serum antithrombin (AT), which binds to heparin sulfate contained in proteoglycans of the glycocalyx, is the key to its anticoagulant effect. Such a combination changes spatial structure of AT. Conformational changes of antithrombin are responsible for its anticoagulant action: they potentiate anticoagulant effect and inhibition of several active coagulation factors [2].
Sulodexide contains $80 \%$ of fast moving heparin (FMH) and $20 \%$ of dermatan sulfate (free fraction) [3]. Structure of $\mathrm{FMH}$ resembles that of unfractionated heparin. Its biological and pharmacological effects on the coagulation cascade are also similar to heparin [3].

Dermatan sulfate contains fewer sulfate residues, thus exhibiting weaker anticoagulant effect. Mean molecular weight of dermatan sulfate (free fraction) is several times greater than $\mathrm{FMH}$. The anticoagulant effect of dermatan sulfate involves inhibition of active coagulation factor II (thrombin) through affinity to heparin cofactor II, which is a natural inhibitor of this factor [4].

Sulodexide is packaged in a parenteral form (ampoules), which can be administered intravenously and intramuscularly, as well as in the form of capsules for oral administration.

Bioavailability of oral preparation of sulodexide reaches $40 \%$ and maximal concentration is reached after four hours from capsule ingestion. Sulodexide exhibits significant affinity to endothelium and glycocalyx - higher than to the circulating proteins (which is why it affects global coagulation parameters only to a minimal extent). When administered orally, it does not affect the activated partial thromboplastin time (aPTT) [5].

Sulodexide is metabolized in the liver and excreted through the kidneys [5]. 
The effect of sulodexide on the coagulation system is less than unfractionated heparin or low molecular weight heparins because its pharmacological action is contained mainly to the glycocalyx.

It should be emphasized that sulodexide accelerates spontaneous fibrinolysis of intravascular thrombi by increasing the concentration of plasminogen activator (tPA) and inhibiting tissue plasminogen inhibitor (PAI) [6]. Sulodexide also reduces platelet aggregation [7].

The anti-inflammatory action of sulodexide constitutes an important biological effect directly related to its clinical effects [7]. It is related directly to the inhibition of thrombin activity and thrombogenesis and indirectly to the inhibition of leukocyte activity and their adhesion to endothelium, reduction of cytokine release and platelet aggregation [7]. Sulodexide exhibits an inhibitory effect on the macrophage, heparanase and metalloproteinase activity ]8].

However, the most important and central effect of sulodexide involves the protection and repair of endothelium and glycocalyx by supplying glycosaminoglycans to its structures [8].

It should be mentioned that sulodexide also affects lipid metabolism. It reduces triglyceride levels and increases HDL concentrations. Sulodexide also reduces blood viscosity [8].

The half-life of sulodexide is significantly longer than in unfractionated heparin. In contrast to heparins, oral administration is feasible [5].

The most important are the biological effects of sulodexide, which translate into clinical effects [I-5]. These effects include anticoagulant action within arteries and veins, profibrinolytic action, anti-inflammatory effect, protection of the endothelium and glycocalyx, and regulation of the vascular tone [8].

\section{Effectiveness and safety of sulodexide in the prevention of venous thromboembolic disease (VTD)}

Scientific evidence regarding the effectiveness and excellent safety profile of sulodexide in the prophylaxis (therapy) of deep vein thrombosis (DVT) recurrence is solid and translated into recommendations contained in the Polish guidelines [9-I2].

The treatment of DVT consists of three phases: acute treatment phase, usually lasting 5-7 days (in patients treated with rivaroxaban this phase lasted 2 I days), long-term anticoagulation therapy lasting 3 months, and chronic prophylaxis (therapy) of DVT recurrence, which lasts from several months to years, and may be indefinite in some patients [12].

Long-term, chronic anticoagulation prophylaxis (therapy) is indicated i.a. in patients with idiopathic DVT.
In this group of patients, the risk of recurrence is only reduced when chronic prophylaxis (therapy) of DVT recurrence with anticoagulants is continued. The risk of DVT recurrence increases when anticoagulation stops and the probability of DVT recurrence is independent of the length of anticoagulation therapy [12].

Two elements play a fundamental role in chronic prophylaxis (therapy) of DVT recurrence: effectiveness of anticoagulant agents measured as reduction in relative risk of DVT recurrence and safety of therapy, which is measured by the incidence of hemorrhagic complications, the most important of which are major bleedings requiring hospitalization - some of them being life-threatening. Sulodexide is characterized by great safety in the prophylaxis of DVT recurrence [II-I3].

To a physician involved in the treatment of DVT, episodes of DVT recurrence, as well as, hemorrhagic complications constitute the most undesirable and critical elements of therapy. Therefore, the incidence of recurrences and the frequency of bleeding complications should be assessed simultaneously as a clinical net benefit when comparing different anticoagulation agents [13]. Unilateral comparison of the effectiveness or safety alone is flawed and may pose a threat to the patient's outcome. Somewhat lower effectiveness of sulodexide in the prevention of DVT recurrence is compensated by an excellent safety profile [13].

Very high effectiveness and excellent safety profile of sulodexide in the prevention of DVT recurrence was demonstrated in the SURVET trial [I I]. It was a multi-center, randomized, double-blind study comparing the effectiveness and safety of sulodexide versus placebo. The study included 615 patients who completed 3-12 months of chronic anticoagulation therapy after an episode of DVT or pulmonary embolism (PE). Deep vein thrombosis was diagnosed in $92 \%$ and $P E$ in $8 \%$ of patients included in the study.

Sulodexide $2 \times 2$ capsules $(2 \times 500$ LSU) or placebo were administered over a 2-year period together with compression therapy.

The effectiveness of sulodexide, basing on the frequency of DVT recurrence and safety, was assessed as the frequency of hemorrhagic complications - major bleeding and clinically relevant bleeding complications other than major.

DVT recurrence took place in $4.9 \%$ of patients in the sulodexide group and in $9.7 \%$ of patients in the placebo group (HR 0.49; 95\% Cl 0.27-0.92; $\mathrm{P}=0.02$; after applying statistical methods of unification of population $\mathrm{HR}=0.45$ ) within a 24-month follow-up period.

There were no major bleeding complications in the group of patients receiving sulodexide as well as in the placebo group. Only two clinically significant bleeding complications other than major bleeding were observed in each group. 
The SURVET trial corroborated an acceptable effectiveness of sulodexide in prevention of VTE recurrence among patients with DVT as well as an excellent therapeutic safety. Obtained results constitute the basis for recommending sulodexide in patients with a high risk of bleeding complications during DVT prophylaxis (therapy) [II, 12].

The two following studies assessing the effectiveness of sulodexide in preventing DVT recurrence are of lesser clinical significance $[9,10]$.

The first one is a registry based on an analysis of 405 patients diagnosed with DVT who have completed 6 months of anticoagulation therapy. Subsequently, patients were allocated to the control group (no anticoagulation) and the group where patients received sulodexide $(2 \times$ I capsule) for 24 months. Both groups did not differ with regard to demographics and presented a similar DVT location profile. The frequency of DVT recurrence reached $7.4 \%$ in the group receiving sulodexide and $17.9 \%$ in the control group $(P<0.05)$ [9].

In another study, the effectiveness and safety of sulodexide was compared with vitamin $\mathrm{K}$ antagonists. The trial included I 50 patients with proximal DVT. After the acute phase of therapy (patients received nadroparin and urokinase) patients were randomly assigned to one of 2 groups. In the first group acenocoumarin was used to keep INR in a range $2-3$, while the other group received sulodexide. Follow-up continued for 3 months. No statistically significant differences were noted with regard to the incidence of VTE recurrence, while the frequency of bleeding complications was significantly higher in the group of patients receiving acenocoumarin (I3\% vs. $0 \% ; P=0.014)$ [10].

While summarizing the effectiveness and safety of sulodexide in prevention of VTE recurrence, it should be emphasized that this agent is characterized by excellent safety and acceptable efficacy.

\section{Administration of sulodexide in chronic venous disease}

CVD is inherently associated with venous insufficiency, which constitutes the pathophysiological basis for the development of clinical manifestations of this disease: asymptomatic venous hypertension, varicose veins of the lower limbs, post-thrombotic syndrome and leg ulcers.

Venous hypertension developed as a result of reflux due to underlying structural and functional abnormalities of the venous system plays a key role in the development of chronic venous insufficiency. Venous hypertension leads to dilatation of veins and development of insufficiency of vein valves. Hemodynamic changes are transferred from macrocirculation to mi- crocirculation. Increase in venous pressures is accompanied by changes in blood flow, disruption of laminar flow and leukocyte adhesion to vascular endothelium, as well as augmented release of proteolytic enzymes. Activated neutrophils and monocytes are sequestered in the vascular endothelium and neighboring tissues. As a result of the above-described mechanisms there is an increased release of inflammatory mediators: chemokines, cytokines, matrix metalloproteinases (MMPs), or transforming growth factor $B$, from activated cells, contributing to the development and progression of inflammation. Chronic inflammation results in destruction of extracellular matrix and irreversible remodeling of venous vessels and valves. Disease progresses as a consequence of these processes, leading to the development of varicose veins and, in the long term, formation of skin lesions and ulcerations $[14,15]$.

Sulodexide exhibits antiproteolytic effect by attenuating the activity of metalloproteinases that are involved in extracellular matrix degradation and play a key role in the processes responsible for the development of chronic venous insufficiency. MMP activity is weakened by inhibition of proteolytic action and reduction of synthesis. Sulodexide was also shown to inhibit the activity of macrophages by reducing the release of inflammatory mediators, such as interleukin- $\mid B$, interleukin-7, interleukin-8, interleukin-12, interleukin-17, MMP-9, and granulocyte and macrophage colony-stimulating factor (GM-CSF). In non-healing ulcers the acute phase markers hinder progression to the granulation phase and ulcer healing. Sulodexide decreases the expression of acute phase proteins in the ulcer, at the same time increasing the expression of granulation phase markers [14].

Literature concerning clinical effects of VTE treatment with sulodexide is quite abundant and the results are consistent [16-23].

Two studies were devoted to the effect of sulodexide on venous hypertension $[16,17]$.

One of them examined changes in microcirculation using capillary filtration coefficient measured with strain gauge pletysmography [16]. Thirty-six patients with VTE were included in the study and randomly allocated to the sulodexide or placebo group. After 30 and 45 days of therapy with sulodexide capillary filtration coefficient was significantly reduced by almost half compared to baseline values, suggesting clinical efficacy of this agent in the treatment of microcirculation disorders in the course of chronic venous insufficiency [16].

In another trial, 476 patients with CVD were analyzed using double-blind randomization [17]. Half of the patients were diagnosed with post-thrombotic syndrome and the other half had idiopathic varicose veins of the lower limbs. Patients were divided into 
three groups with equal distribution of different forms of CVD. In the first group sulodexide in a daily dose of $2 \times$ I capsule $(50 \mathrm{mg})$ was used, in the second $-2 \times$ 2 capsules $(2 \times 50 \mathrm{mg})$, and in the third group $-1 \times$ 4 capsules $(I \times 100 \mathrm{mg})$ for 60 days. Effects of therapy were controlled by Doppler phlebotensiometry, which was performed before commencement of treatment, after I month and after 2 months. Venous pressure was measured in upright and supine position. Measurements were performed on the great saphenous vein and posterior fibular vein. Therapeutic response (reduction in venous pressure) depended on the dose of sulodexide - the greatest response was noted in the group receiving 4 capsules (100 mg) per day regardless of whether it was given in a single dose or in two doses. Side effects in the form of gastrointestinal disorders were observed in 10\% of patients included in the study - these symptoms subsided after 72 hours and did not require discontinuation of treatment [17].

The goal of another four randomized studies was to assess the effectiveness of sulodexide in the treatment of venous ulcers [ $18-2 \mid]$. Compression therapy was also used in all of those studies.

In the first trial, the effectiveness of sulodexide in the treatment of venous ulcers larger than $2 \mathrm{~cm}$ was assessed using a double-blind technique [18]. Two hundred and thirty-five patients were included. Greater frequency of complete ulcer healing was observed in the group receiving sulodexide after 2 months of treatment compared to the control group (35\% vs. $20.9 ; P=0.018)$ and after 3 months (52.5\% vs. $32.7 \%$; $P=0.004)$. Adverse effects occurred in $19 \%$ of patients in the sulodexide group, only 4 of which were considered related to the drug [18].

In another study assessing the frequency of total ulcer healing after 2 months, results were based on the analysis of 94 patients [19]. In the sulodexide group healing was demonstrated in $58 \%$ of subjects compared to $36 \%$ in the placebo group $(P=0.03)$ [19].

In an analysis performed by Kucharzewski et al. the efficacy of sulodexide was demonstrated in the treatment of large venous ulcers (mean ulcer surface was $12.4 \mathrm{~cm}^{2}$ ) [20]. The study included 44 patients. After 7 weeks of sulodexide administration recovery was observed in $70 \%$ compared to $35 \%$ in the control group $(P<0.05)[20]$.

The last trial from this group was based on the analysis of I I4 patients [2I]. Ulcer healing was evaluated after a month of sulodexide use. Complete remission after 30 days was observed in $52.5 \%$ of patients from sulodexide group vs. $32 \%$ from the control group $(\mathrm{P}<0.05)[2 \mathrm{I}]$.

A meta-analysis of the above-mentioned studies showed that the NNT (number needed to treat) for complete healing was 4 [22].
Consistent results of the presented studies gained sulodexide a place in the recommendations of numerous scientific societies (American College of chest Physicians, American Venous Forum and Society for Vascular Surgery) for pharmacological treatment of venous ulcers. Sulodexide has a high level of evidence for the treatment of venous ulcers (GRADE A) according to the guidelines for the management of chronic venous lower limb disorders published in the International Angiology in 2018 [23].

The utility of sulodexide in the prophylaxis of post-thrombotic syndrome was also evaluated based on a registry of 339 patients [24]. The incidence of the post-thrombotic syndrome after 60 months was lower in the group receiving sulodexide (12.2\% vs. $19.5 \%$ in the standard treatment group and $12.2 \%$ vs. $23.5 \%$ in the aspirin group; $\mathrm{P}<0.05$ for both analyses).

It should be emphasized that sulodexide is registered for the treatment of symptomatic primary or secondary venous insufficiency. In a study encompassing 450 patients with CVD evaluating the effects of the drug at a dose of $2 \times 1$ capsule/day for 3 months on objective and subjective symptoms as well as on the quality of life of studied subjects a statistically significant effect of the drug on: pain, redness, skin thickening, skin temperature, feeling of heavy legs, paresthesia, cramps, was demonstrated. The examined symptoms became reduced by over $60 \%(P<0.0001)$. Also, the quality of life measured using CIVIQ survey was significantly improved [25].

In the previously cited clinical trial of 476 patients the effect of the drug on the attenuation of CVD symptoms, including reduction of leg edema, was demonstrated [17]. The Society for Vascular Surgery and the American Venous Forum propose use of venoactive agents, including sulodexide, in addition to compression therapy in the treatment of patients with pain and edema due to CVD.

In the conclusion of the section devoted to CVD the proven efficacy and safety of sulodexide in the treatment of venous ulcers and symptoms of CVD should be once again emphasized. Prophylaxis of post-thrombotic syndrome is one of the areas of the potential application of this drug [24].

Two studies demonstrated that sulodexide inhibits growth factors responsible for fibrosis and angiogenesis [VEGF (vascular endothelial growth factor), TGF-beta (transforming growth factor)] [26, 27].

\section{Conclusions}

Based on the review of the literature we assessed the effectiveness and safety of sulodexide in the prophylaxis of deep vein thrombosis recurrence and treatment 
of chronic venous disease. Sulodexide was proven effective and safe in preventing recurrence of deep vein thrombosis and effective in the treatment of venous ulcers as well as other forms of CVD. An immanent feature of sulodexide - very low frequency of hemorrhagic complications, should be especially emphasized.

\section{Conflict of interest}

None.

\section{References:}

I. Zhang X, Sun D, Song JW, et al. Endothelial cell dysfunction and glycocalyx - A vicious circle. Matrix Biol. 2018; 71-72: 42I-43I, doi: 10.1016/j.matbio.2018.01.026, indexed in Pubmed: 29408548.

2. Rosenberg RD, Shworak NW, Liu J, et al. Heparan sulfate proteoglycans of the cardiovascular system. Specific structures emerge but how is synthesis regulated? J Clin Invest. 1997; 99(9): 2062-2070, doi: I0. I I72/JCII I9377, indexed in Pubmed: 9151776.

3. Veraldi N, Guerrini M, Urso E, et al. Fine structural characterization of sulodexide. J Pharm Biomed Anal. 2018; 156: 67-79, doi: 10.1016/j.jpba.2018.04.012, indexed in Pubmed: 29689470.

4. Tollefsen DM, He Li, Giri TK, et al. Vascular dermatan sulphate and heparin cofac-tor II. Prog Mol Biol Transl Sci. 2010; 93(I): 35I-372.

5. Marchi E, Barbanti M, Milani R, et al. Organ glycosaminoglycan distribution after intravenous and oral administration in rats. Semin Thromb Hemost. 1994; 20(3): 297-300, doi: 10.1055/s2007-1001916, indexed in Pubmed: 7824965.

6. Mauro M, Ferraro G, Palmieri GC. Profibrinolytic and antithrombotic effects of sulodexide oral administration: a double-blind, cross-over, placebo-controlled study. Curr Ther Res. 1992; 51: 342-50.

7. Cerletti C, Rajtar G, Marchi E, et al. Interaction between glycosaminoglycans, platelets, and leukocytes. Semin Thromb Hemost. 1994; 20(3): 245-253, doi: 10.1055/s-2007-1001909, indexed in Pubmed: 7824958.

8. Carroll BJ, Piazza G, Goldhaber SZ. Sulodexide in venous disease. J Thromb Haemost. 2019; 17(1): 31-38, doi: 10.1 III/ jth. 14324, indexed in Pubmed: 30394690.

9. Errichi BM, Cesarone MR, Belcaro G, et al. Prevention of recurrent deep venous thrombosis with sulodexide: the SanVal registry. Angiology. 2004; 55(3): 243-249, doi: $10.1177 / 000331970405500302$, indexed in Pubmed: 15156256.

10. Cirujeda JL, Granado PC. A study on the safety, efficacy, and efficiency of sulodexide compared with acenocoumarol in secondary prophylaxis in patients with deep venous thrombosis. Angiology. 2006; 57(I): 53-64, doi: 10.1 177/000331970605700108, indexed in Pubmed: 16444457.

I I. Andreozzi GM, Bignamini AA, Davì G, et al. SURVET Study Investigators. Sulodexide for the Prevention of Recurrent Venous Thromboembolism: The Sulodexide in Secondary Prevention of Recurrent Deep Vein Thrombosis (SURVET) Study: A Multicenter, Randomized, Double-Blind, Placebo-Controlled Trial. Circulation. 2015; 132(20): 1891-1897, doi: 10.1161/CIRCULATIONAHA.I I5.016930, indexed in Pubmed: 26408273.
12. Tomkowski W, Kuca P, Urbanek T, et al. Venous thromboembolism - recommendations on the prevention, diagnostic approach and management. The 2017 Polish Consensus Statement. Acta Angiologica. 2017; 23(2): 35-7I, doi: 10.5603/aa.2017.0008.

13. Tomkowski W, Kuca P, Bignamini AA, et al. Extended use of sulodexide, apixaban, rivaroxaban and dabigatran in venous thromboembolism: indirect comparison of clinical trials. Int Angiol. 2017; 36(5): 496-497, doi: 10.23736/S0392-9590.17.03819-6, indexed in Pubmed: 2864l 408.

14. Carroll BJ, Piazza G, Goldhaber SZ. Sulodexide in venous disease. J Thromb Haemost. 2019; 17(I): 31-38, doi: 10.11।1/ jth. 14324 , indexed in Pubmed: 30394690.

15. Andreozzi GM. Sulodexide in the treatment of chronic venous disease. Am J Cardiovasc Drugs. 2012; 12(2): 73-8I, doi: 10.2165/11599360-000000000-00000, indexed in Pubmed: 22329592.

16. Cospite M, Ferrara F, Cospite V, et al. Sulodexide and the microcirculatory component in microphlebopathies. Curr Med Res Opin. 1992; 13(I): 56-60, doi: 10.1185/03007999209।15223 , indexed in Pubmed: 1468246.

17. Saviano M, Maleti O, Liguori L. Double-blind, double-dummy, randomized, multi-centre clinical assessment of the efficacy, tolerability and dose-effect relationship of sulodexide in chronic venous insufficiency. Curr Med Res Opin. 1993; 13(2): 96-108, doi: 10.1 |85/03007999309| I I 538 , indexed in Pubmed: 8325047.

18. Coccheri S. Randomized, double-blind, multicentre, placebo controlled study of sulodexide in the treatment of venous leg ulcers. Thromb Haemost. 2002; 87: 947-952.

19. Scondotto G, Aloisi D, Ferrari P, et al. Treatment of venous leg ulcers with sulodexide. Angiology. 1999; 50(II): 883-889, doi: 10. I I77/00033 197990500 I 102, indexed in Pubmed: 10580352.

20. Kucharzewski M, Franek A, Koziolek $H$. Treatment of venous leg ulcers with sulodexide. Phlebologie. 2003; 32(II5): 120.

21. Zhou YX, Feng $X$, Jing ZP. Efficacy and safety of sulodexide in the treatment of venous ulcers of leg. PharmCare Res. 2007; 7: $22-24$.

22. Nelson EA, Jones J. Venous leg ulcers. BMJ Clin Evid. 2008: 1902-1937.

23. Nicolaides A, Kakkos S, Baekgaard N, et al. Management of chronic venous disorders of the lower limbs: guidelines according to scientific evidence. Int Angiol. 2008; 27(I): I-59, indexed in Pubmed: 18277340.

24. Luzzi R, Belcaro G, Dugall M, et al. The efficacy of sulodexide in the prevention of postthrombotic syndrome. Clin Appl Thromb Hemost. 2014; 20(6): 594-599, doi: 10.1 | 77// 0760296 |4533 |43, indexed in Pubmed: 2478/ 035.

25. Elleuch N, Zidi H, Bellamine Z, et al. CVD study investigators. Sulodexide in Patients with Chronic Venous Disease of the Lower Limbs: Clinical Efficacy and Impact on Quality of Life. Adv Ther. 2016; 33(9): 1536-1549, doi: 10.1007/s | 2325-016-03599, indexed in Pubmed: 27397587.

26. Borawski J, Dubowski M, Pawlak K, et al. Sulodexide induces hepatocyte growth factor release in humans. Eur J Pharmacol. 2007; 558(I-3): 167-17I, doi: 10.1016/j.ejphar.2006.12.009, indexed in Pubmed: 17258198.

27. Pletinck A, Van Landschoot M, Steppan S, et al. Oral supplementation with sulodexide inhibits neo-angiogenesis in a rat model of peritoneal perfusion. Nephrol Dial Transplant. 2012; 27(2): 548-556, doi: 10.1093/ndt/gfr370, indexed in Pubmed: 21750165 . 